\title{
La Contribución de PROMIPAC Zamorano al Manejo Integrado de Plagas en América Central
}

\author{
Alfredo Rueda ${ }^{1}$, Orlando Cáceres ${ }^{2}$, Julio López ${ }^{3}$, Ernesto Garay ${ }^{4}$ y Yordana Valenzuela ${ }^{5}$
}

\begin{abstract}
Resumen. El Programa Manejo Integrado de Plagas en América Central (PROMIPAC) ejecutado por la Escuela Agrícola Panamericana, con fondos de la Agencia Suiza para la Cooperación y el Desarrollo, se realizó de 1995 a 2012 en Honduras, Nicaragua y El Salvador. El objetivo del programa fue contribuir al desarrollo agrícola de la región a través de la promoción del Manejo Integrado de Plagas (MIP). El programa fortaleció las acciones en extensión, educación e investigación de 80 instituciones socias, incluyendo organizaciones gubernamentales, no gubernamentales, educativas y privadas. El programa generó información técnica, que incluyó la producción de decenas de documentos, entre manuales, cartillas, libros, videos y tesis. PROMIPAC fue pionero en la implementación de la metodología de Escuelas de Campo, realizando 425 en hortalizas y granos básicos. El programa capacitó a 2,860 técnicos extensionistas, 1,830 promotores, 729 docentes y 2,382 estudiantes. Los técnicos capacitaron a 37,167 agricultores/as que adoptaron más de 40 tecnologías MIP y obtuvieron un ingreso incremental anual estimado de 300 US dólares por persona.
\end{abstract}

Palabras clave: COSUDE, Escuelas de Campo, extensión, educación, investigación, MIP.

\section{Contribution of PROMIPAC Zamorano to Integrated Pest Management in Central America}

\begin{abstract}
The Integrated Pest Management Program in Central America (PROMIPAC) implemented by the Pan-American Agricultural School and funded by the Swiss Agency for Cooperation and Development, was conducted from 1995 to 2012 in Honduras, Nicaragua and El Salvador. The objective of the program was to contribute to agricultural development in the region through the promotion of Integrated Pest Management (IPM). The program strengthened the extension, education and research capabilities of 80 partner institutions, including government, non-governmental, educational and private agencies. The program generated technical information which included dozens of documents, such as manuals, booklets, books, videos and thesis. PROMIPAC pioneered the implementation of the Farmer Field School methodology, having done 425 in vegetables and grains. The program technicians trained 2,860 extension agents, 1,830 promoters, 729 teachers and 2,382 students. The technicians trained 37,167 farmers that adopted more than 40 IPM technologies, which resulted in a US \$ 300 per person increase in estimated annual income.
\end{abstract}

Keywords: Education, Extension, Farmer Field Schools, Research, SDC.

\section{Antecedentes}

Los programas de Manejo Integrado de Plagas (MIP) de Zamorano, que iniciaron en 1983 con fondos de USAID, fueron pioneros en trabajar en proyección, investigación y extensión con productores de granos básicos en Honduras. Posteriormente se trabajó con vegetales como cebolla, melón y repollo en Honduras, Nicaragua y Guatemala. Estos programas MIP tenían como objetivo investigar el efecto de las plagas en los cultivos y generar tecnologías MIP para su control.
Adicionalmente, se trabajó con modelos de enseñanzaaprendizaje para productores, probando varios métodos para la entrega de los contenidos técnicos. Los programas MIP de Zamorano se distinguieron por realizar investigación en campo, fomentar la participación activa de los productores en el desarrollo y validación de tecnologías, y considerar aspectos socioeconómicos de los productores en el desarrollo de menús tecnológicos MIP.

A lo interior de Zamorano, los programas MIP institucionalizaron la fitoprotección con la creación

\footnotetext{
${ }^{1}$ Excoordinador Regional PROMIPAC, Zamorano, alfredorueda@yahoo.com

${ }^{2}$ Excoordinador Nacional El Salvador PROMIPAC, Zamorano, caceresorlando@ hotmail.com

${ }^{3}$ Coordinador Regional PROMIPAC, Zamorano, jlopez@zamorano.edu

${ }^{4}$ Excoordinador Nacional Honduras PROMIPAC, Zamorano, ejgaray@ hotmail.cm

${ }^{5}$ Excoordinadora Monitoreo y Evaluación PROMIPAC, Zamorano, yordanavalenzuela@ hotmail.com
} 
del Departamento de Protección Vegetal (DPV), el cual ofrecía un programa de ingeniería agronómica con especialización de dos años en protección vegetal, un año de clases y otro de trabajo en proyección y/o investigación en el campo. El DPV contaba con un equipo de especialistas en fitoprotección y unidades operativas como El Centro de Evaluación de Plaguicidas (CEMPLA) que evaluaba plaguicidas y bioplaguicidas para medir la eficacia como requisito para su registro, y ofrecía entrenamiento en métodos de aplicación de plaguicidas, plaguicidas botánicos, y en Manejo Racional de Plagas y Plaguicidas. El DPV también contaba con el Laboratorio de Control Biológico el cual tenía una unidad de cuarentena para la importación de enemigos naturales para control biológico clásico, el laboratorio de Diagnóstico en Fitoprotección y la Colección Entomológica, una de las mejores de la región centroamericana. Para el aprendizaje, el DPV estableció y mantenía una colección viva de malezas, plantas medicinales y plantas con propiedades de plaguicidas botánicos.

En 1990, Zamorano ejecutó el programa MIP Laderas financiado al inicio por el Fondo Contravalor Honduras-Suiza y luego por PNUD Honduras. Este programa apoyó en MIP a pequeños productores de laderas atendidos por Vecinos Mundiales, Finca Loma Linda, el Centro Internacional de Desarrollo e Investigación en Cultivos de Cobertura (CIDICCO), Catholic Relief Services (CRS) y la Secretaria de Agricultura y Ganadería (SAG) del Gobierno. El programa tenía por objetivo generar capacidades en MIP dentro de las instituciones. Al tratarse de pequeños productores de subsistencia en laderas los enfoques fueron orientados a la promoción de control biológico natural y uso de productos botánicos para controlar las plagas. Este proyecto fue para Zamorano el aprendizaje para trabajar como programa de segundo piso y en consorcio con otros socios al mismo nivel de participación.

\section{Inicios del Programa}

En 1994, la Cooperación Suiza (COSUDE) y la Escuela Agrícola Panamericana, Zamorano, Honduras, establecieron un convenio de colaboración para desarrollar un Programa MIP en Nicaragua para conjugar esfuerzos para el mejoramiento de la calidad de vida de los pequeños productores de granos básicos. La Cooperación Suiza estaba desarrollando programas agrícolas en Nicaragua, tales como FOMENTA, POSTCOSECHA, PASOLAC y MIP con INTA, y consideraban que los programas MIP de Zamorano reforzarian tecnológicamente a los programas de extensión e investigación del gobierno de Nicaragua

El programa se fundamentó en la reducción del uso de plaguicidas usados de forma indiscriminada para el control de las plagas de cultivos, que causan efectos negativos en el medio ambiente y la salud humana. La información estadística reveló que en el período de 1994-1999 la importación de plaguicidas en Nicaragua aumentó de 21.7 a 49.3 millones de dólares, convirtiéndose en una preocupación por el uso de plaguicidas de alta toxicidad. Los plaguicidas en esa época eran subsidiados con el fin de incrementar la producción de granos básicos. Esto trajo como consecuencia que los productores usaban indiscriminadamente los plaguicidas ya que tenían acceso fácil y barato, sin tener el conocimiento de cuando aplicar y cómo manejarlos de forma segura. Estadísticas del Programa de Plaguicidas del Ministerio de Salud y del Boletín Epidemiológico de PLAGSALUD indican que en 1997 se registraron 1429 casos de intoxicaciones agudas por plaguicidas en diez departamentos del país. El número de personas atendidas en hospitales nicaragüenses por intoxicaciones con plaguicidas era alarmante.

\section{Fases del Programa}

El programa MIP de la Cooperación Suiza ejecutado por Zamorano se implementó de 1994 al 2012. El programa se dividió en fases de tres a cuatro años de duración. Al finalizar cada fase se realizaba una evaluación exhaustiva y una planificación participativa con los socios del programa para elaborar los objetivos y metas de la nueva fase.

El programa se denominó en un inicio MIP/ZAMORANO/COSUDE, tenía sede en las instalaciones del laboratorio de diagnóstico de plagas del MAGFOR en Nicaragua compartiendo instalaciones con un programa MIP para café y repollo del CATIE. MIP/ZAMORANO/COSUDE concentraba sus esfuerzos en granos básicos, ajonjolí y algunas 
hortalizas para mercados nacionales. Paralelamente, el INTA mantenía otro programa MIP financiado por COSUDE que implementaba por medio de sus extensionistas. MIP/ZAMORANO/COSUDE hizo un mapeo participativo con agricultores, universidades, ONG y gobierno para tener una visión de la realidad en el campo de la utilización del MIP en el país y de los requerimientos institucionales para obtener una mayor aplicación del mismo en Nicaragua.

Primera fase (1995-1998). Se desarrolló en Nicaragua y su estrategia de implementación se enmarcó en la articulación de organizaciones gubernamentales, universidades, organizaciones no gubernamentales y privadas para juntar esfuerzos en un proceso de fortalecimiento para apoyar a los productores de granos básicos, en el desarrollo de acciones de MIP, como contribución a la sostenibilidad de los sistemas agrícolas.

Segunda fase (1998-2002). Se extendieron las operaciones a El Salvador aplicando las experiencias ganadas en Nicaragua. Se cambió el nombre a Programa de Manejo Integrado de Plagas en América Central (PROMIPAC) para darle visibilidad propia al programa. Se implementó una nueva estrategia para el trabajo en asocio, que consistió en crear una bolsa competitiva de proyectos, donde las instituciones interesadas tuvieran acceso a recursos y asesoría técnica para implementar el MIP con productores.

Tercera fase (2002-2004). El programa continuó trabajando en Nicaragua y El Salvador, se concentraron esfuerzos en introducir, validar y adaptar una metodología de extensión basada en la educación de adultos, llamada Escuelas de Campo para Agricultores (ECA) con el fin de tener una herramienta metodológica adaptada al medio, con la cual se pudiera obtener más adopción del MIP.

Cuarta fase (2005-2008). Se profundizaron las actividades en Nicaragua, se cerró el programa en El Salvador y se iniciaron actividades de campo en Honduras. En esta etapa, PROMIPAC ya contaba con un grado de madurez de los equipos técnicos en los tres países. Las actividades se realizaron como un proyecto de segundo piso encargado de fortalecer las capacidades técnicas y metodológicas de instituciones de desarrollo, ONG públicas, educativas, asociaciones de productores y cooperativas. La gobernanza del programa se dirigió por medio de un comité consultivo regional en el cual participaban los ministerios de agricultura, ONG, sector privado, COSUDE y Zamorano. Los mensajes MIP se extendieron masivamente mediante la aplicación de las ECA manejadas por las instituciones socias.

Quinta fase (2009-2012). Se trabajó en Nicaragua y Honduras concentrando actividades en regiones donde el programa podría tener mayor impacto. Se consolidaron las actividades y se realizó un esfuerzo de fortalecer referentes nacionales en las aéreas estratégicas del programa con el fin de que al terminar el programa las actividades MIP continúen.

\section{Áreas de Acción}

Durante la vida del programa se dividieron las actividades en tres grandes áreas estratégicas: Extensión, Educación, e Investigación. Adicionalmente, el programa incluía las áreas trasversales de género, comunicación, gestión de conocimiento, incidencia en políticas, monitoreo y evaluación, y administración.

Extensión. Comprendió actividades dirigidas a que los productores adoptaran prácticas MIP en sus cultivos. Incluyó actividades de capacitación y formación en MIP, Manejo Integrado de Cultivos (MIC) y metodologías de extensión como las ECA de técnicos, promotores y agricultores. También cubrieron acciones para que los socios nacionales se fortalecieran para ser referentes MIP a nivel nacional.

Educación. Contribuyó con actividades dirigidas a que estudiantes de agricultura de universidades, escuelas técnicas, bachilleratos y escuelas medias y primarias, aprendieran y practicaran MIP, MIC y metodologías de extensión. Se formaron grupos de instituciones educativas para incluir en los currículos y pensum académicos contenidos MIP relevantes para la formación de profesionales, enfatizando en el Aprender-Haciendo. 
Investigación. Se trabajó en generar y validar información sobre temas emergentes de fitoprotección relevantes para los productores. Se trabajó en mejorar las capacidades de investigadores, estudiantes y agricultores. Se trabajó en investigación formal con investigadores y estudiantes, y en investigación campesina o participativa con agricultores $\mathrm{y}$ promotores agrícolas.

\section{Extensión}

En la primera fase del programa la estrategia de implementación se enmarcó en fortalecer los equipos técnicos de las instituciones socias para mejorar la implementación del MIP en el campo. Se realizaron alianzas con organizaciones gubernamentales, universidades y organizaciones no gubernamentales para juntar esfuerzos en un proceso de fortalecimiento colectivo para apoyar a los productores de granos básicos, en el desarrollo de acciones MIP.

Se establecieron convenios de colaboración con el Instituto Nacional de Tecnología Agropecuaria (INTA), el Programa Campesino a Campesino de la Unión Nacional de Agricultores y Ganaderos (UNAG), y otras organizaciones no gubernamentales que desarrollaban proyectos productivos con pequeños agricultores en las regiones I y II de Nicaragua. Al final de la primera fase el proyecto tenía alianzas para la implementación del MIP con 14 instituciones.

Se fortalecieron los conocimientos de los extensionistas sobre fitoprotección capacitándolos con especialistas de la Escuela Agrícola Panamericana y de universidades de Nicaragua. Se organizaron grupos de especialistas MIP por rubro para apoyar las capacitaciones. Se generó información sobre alternativas de manejo y manuales para el control de plagas del maíz, frijol y ajonjolí. Se organizaron foros y se elaboraron manuales sobre plaguicidas botánicos, género y MIP. Para la promoción del MIP en el país, se organizaron Grupos Interinstitucionales Regionales MIP. En esta fase se puso énfasis en agroecología y prácticas ecológicas para reducir los ataques de plagas y las aplicaciones de plaguicidas.

En la segunda fase se ofreció entrenamiento en servicio y capacitación a extensionistas de las instituciones socias. Los grupos de especialistas produjeron material técnico para las capacitaciones. Se realizaron congresos regionales MIP en Nicaragua y El Salvador para compartir tecnologías y conocer los avances en MIP. Se continuó el trabajo de extensión en asocio con las instituciones gubernamentales y no gubernamentales que brindaban asistencia técnica y capacitación a productores de granos básicos y oleaginosas. Se fortalecieron en MIP los equipos técnicos de INTA en Nicaragua y CENTA en El Salvador y de organismos no gubernamentales y privados.

Se consolidaron los grupos regionales que hacian talleres y jornadas de reforzamiento para los técnicos. Los grupos de especialistas estuvieron activos capacitando, elaborando documentos y asesorando parcelas demostrativas en las que se implementaron tecnologías MIP. Los extensionistas capacitados transmitieron las tecnologías MIP a 5000 productores de Nicaragua y El Salvador.

En la tercera fase, el énfasis fue mejorar la calidad de la transferencia del MIP. Se promovió en Nicaragua y El Salvador la metodología de extensión grupal basada en la educación de adultos, llamada Escuelas de Campo (ECA). Los principios fundamentales de las ECA son: el campo en la mejor fuente de aprendizaje, la curricula de capacitación es definida basada en el diagnóstico de la problemática de los cultivos y a las necesidades de capacitación de los productores, y la capacitación cubre todo el ciclo del cultivo. PROMIPAC fue pionero en introducir, adaptar y promover la metodología de ECA en Honduras, Nicaragua y El Salvador.

El análisis agroecológico (AAE) es la principal herramienta para tomar decisiones sobre el manejo de plagas y de cultivo en la ECA. El AAE consiste en analizar las relaciones ecológicas entre insectos (plagas y benéficos), clima y el cultivo, para determinar las acciones a seguir.

El proyecto amplió su cobertura de cultivos y se enfocó en el manejo de plagas de hortalizas. En parcelas demostrativas se implementaron tecnologías MIP para que los productores pudieran valorar la efectividad de las mismas. Con la inclusión de las hortalizas, el programa comenzó a desarrollar en las curriculas temas de organización y mercadeo.

Los niveles de conocimientos del MIP de los productores aumentaron en $40 \%$. Más de la mitad de los técnicos entrenados implementaron la metodología 
Rueda et al.: La Contribución de PROMIPAC Zamorano al Manejo Integrado de Plagas en América Central

ECA en sus instituciones y un tercio de las instituciones socias adoptaron la metodología. Más de la mitad de los agricultores capacitados, implementaron prácticas alternativas y redujeron las aplicaciones de plaguicidas.

En la cuarta fase, debido a las tendencias y demandas de los mercados nacionales, regionales e internacionales en la utilización de Buenas Prácticas Agrícolas (BPA), el proyecto comenzó a promover este enfoque en la producción de cultivos. Se creó una red con los socios interesados en promover las BPA. Se compilaron y divulgaron normativas relacionadas al mercado internacional con Globalgap. En las capacitaciones se incluyeron temas de valor agregado y comercialización.

Siguiendo las recomendaciones de evaluadores externos, junto con las instituciones socias se impulsaron métodos masivos de extensión, se transmitieron 311 programas de radio y tres anuncios en televisión y 19 cuñas radiales relacionadas a MIP o BPA. Se organizaron ferias y congresos nacionales en agricultura orgánica, hortalizas, plaguicidas, medio ambiente y cambio climático.

Se capacitaron los productores en el campo a través de la metodología de ECA. Se promovieron 43 tecnologías MIP (Cuadro 1) en parcelas demostrativas para la producción de granos básicos y hortalizas de calidad. Las tecnologías promovidas por PROMIPAC fueron principalmente preventivas para reforzar el principio de que en MIP es mejor prevenir que reaccionar. Se dio un fuerte impulso al control biológico promoviendo bioplaguicidas desarrollados en Zamorano como alternativas de control ambientalmente amigables.

Cuadro 1. Tecnologías de Manejo Integrado de Plagas (MIP) promovidas por PROMIPAC

\begin{tabular}{l|l}
\hline Prácticas MIP & Objetivo de la práctica \\
\hline Presiembra & \\
\hline $\begin{array}{l}\text { Incorporación de rastrojos y eliminación de } \\
\text { plantas hospederas de enfermedades }\end{array}$ & $\begin{array}{l}\text { Prevenir la transmisión de enfermedades transmitidas por } \\
\text { vectores e incorporar materia orgánica al suelo }\end{array}$ \\
\hline Roturación y volteo del suelo & Manejo de semillas de malezas \\
\hline Labranza en seco & Manejo de Cyperus rotundus y plagas de suelo \\
\hline Encalado del suelo & $\begin{array}{l}\text { Regular el pH para manejo de enfermedades y absorción de } \\
\text { nutrientes por las plantas }\end{array}$ \\
\hline Uso de trampas lumínicas & Manejo de adultos de plagas del suelo \\
\hline Siembra e incorporación de abonos verdes & $\begin{array}{l}\text { Aumentar la fertilidad y el contenido de la materia orgánica } \\
\text { del suelo }\end{array}$ \\
\hline $\begin{array}{l}\text { Siembra de cultivos atrayentes de insectos } \\
\text { benéficos }\end{array}$ & Control biológico natural de la fauna insectil de los cultivos \\
\hline Establecimiento de barreras vivas & $\begin{array}{l}\text { Manejo de insectos vectores de enfermedades, erosión de } \\
\text { suelos }\end{array}$ \\
\hline Barreras muertas & Control de la erosión de suelo en laderas \\
\hline Producción de plantas sanas en invernadero & Manejo de enfermedades del suelo y follaje en hortalizas \\
\hline Uso de lombrihumus & $\begin{array}{l}\text { Aumentar el contenido de la materia orgánica y de la } \\
\text { biodiversidad en el suelo }\end{array}$ \\
\hline Sustratos para la producción de plántulas & Manejo de enfermedades del suelo \\
\hline Uso del plástico agrícola (acolchado) & Repelencia a insectos plagas, conservación del agua \\
\hline Riego por goteo & Conservación de agua y manejo de enfermedades \\
\hline Control de malezas & Manejo de plantas hospederas de plagas \\
\hline Control de malezas & Manejo de plantas hospederas de plagas \\
\hline Solarización & Manejo de plagas del suelo en almácigos \\
\hline Biofumigación & Manejo de plagas del suelo en almácigos \\
\hline
\end{tabular}


Cuadro 1. Continuación

\begin{tabular}{|c|c|}
\hline Prácticas MIP & Objetivo de la práctica \\
\hline \multicolumn{2}{|l|}{ Siembra a cosecha } \\
\hline Uso de variedades tolerantes & Manejo de enfermedades \\
\hline Rotación de cultivos & Manejo de enfermedades e insectos \\
\hline Cultivos en asocio & Manejo de enfermedades e insectos plagas \\
\hline $\begin{array}{l}\text { Uso de plaguicida biológico (Trichoderma) en } \\
\text { tratamiento de semillas }\end{array}$ & Manejo de enfermedades del suelo \\
\hline Distancias y densidad de siembra & Manejo de plagas del suelo \\
\hline Siembra en camas o bordos altos & Manejo de enfermedades en hortalizas \\
\hline Siembra en curvas a nivel & Control de erosión del suelo \\
\hline Uso de Beauveria bassiana & Control de plagas del suelo \\
\hline Uso de agua azucarada & Atrayente de enemigos naturales \\
\hline Uso de solución arrancadora (fósforo) & $\begin{array}{l}\text { Manejo de la fertilidad del suelo y desarrollo radicular en } \\
\text { hortalizas. }\end{array}$ \\
\hline Cobertura de cultivo con microtúnel & Manejo de vectores de enfermedades \\
\hline Siembra en macrotúneles & Manejo de vectores de enfermedades \\
\hline Siembra en casa malla & Manejo de vectores de enfermedades \\
\hline $\begin{array}{l}\text { Uso de trampas de colores (amarillas, verdes y } \\
\text { azules) }\end{array}$ & Manejo de insectos vectores de enfermedades \\
\hline $\begin{array}{l}\text { Uso de productos botánicos a base de nim para } \\
\text { el control de plagas }\end{array}$ & Manejo de plagas del follaje \\
\hline Uso del caldo bordelés & Manejo de enfermedades \\
\hline Uso del caldo sulfocálcico & Manejo de enfermedades \\
\hline Uso de plaguicidas de origen botánicos & Manejo de plagas insectiles \\
\hline Uso de reguladores del $\mathrm{pH}$ del agua & Aumentar la efectividad de los plaguicidas \\
\hline Triple lavado del equipo de aplicación & Reducir la intoxicación por plaguicidas \\
\hline Uso de feromonas & Muestreo de insectos plagas \\
\hline Uso de trampas atrayentes (olorosa) & Manejo de adultos de insectos plagas \\
\hline Incorporación de rastrojos & Evitar fuentes de inóculo \\
\hline $\begin{array}{l}\text { Uso de adherente y dispersante en la mezcla de } \\
\text { agroquímicos }\end{array}$ & Aumentar la efectividad del plaguicida \\
\hline \multicolumn{2}{|l|}{ Poscosecha } \\
\hline $\begin{array}{l}\text { Uso de silo metálico para almacenamiento de } \\
\text { granos }\end{array}$ & Manejo de plagas en poscosecha \\
\hline Cosecha temprana & Evitar colonización en maíz con gorgojo \\
\hline
\end{tabular}

Se coordinó con 32 instituciones a las cuales se les fortalecieron los equipos técnicos en MIP y la metodología ECA. Se capacitaron 1,180 productores líderes, promotores o extensionistas comunitarios para que apoyaran la ejecución de actividades de MIP en sus comunidades. La evaluación externa indicó que se logró al final de la fase una adopción de tecnologías MIP de $66 \%$ en El Salvador y $59 \%$ en Nicaragua.
En la quinta fase de PROMIPAC, la estrategia de salida en ambos países fue trasladar la experiencia y conocimientos a las instituciones socias. El programa focalizó los esfuerzos en la formación y consolidación de referentes temáticos, de esta manera se crearon referentes en temas MIP, MIC, BPA, Promotoría y Formación de Especialistas en ECA y Doctores en Plantas, quienes a futuro apoyarán la búsqueda de 
soluciones a los problemas fitosanitarios en el campo.

En la última fase, las ECA se consolidaron como metodología de extensión. La metodología de ECA tiene una amplia aceptación a nivel nacional en los tres países, en el sector gubernamental, instituciones privadas y organismos no gubernamentales. La metodología ha sido adaptada a diversos ambientes y rubros para realizar el trabajo de extensión con productores.

Se desarrollaron programas para fortalecer las capacidades en sanidad vegetal y MIP en los ministerios de agricultura respectivos. Los técnicos del proyecto también capacitaron a técnicos de instituciones socias del sector privado y no gubernamental. Se elaboraron materiales técnicos para promotores, y extensionistas.

Buscando la sostenibilidad de las acciones después de la salida del programa, se diseñó un programa certificado de promotores MIP. Estos promotores adquirieron las competencias para continuar asesorando en MIP a los productores de sus comunidades. Se certificaron 400 promotores y se estableció una curricula para certificación de promotores en Honduras y Nicaragua. Se capacitaron 2,860 técnicos extensionistas y 1,830 promotores. Se realizaron 425 Escuelas de Campo. Como resultado del área de extensión, el programa llegó a 37,167 agricultores que obtuvieron un ingreso incremental anual estimado de 300 dólares por persona.

\section{Educación}

PROMIPAC, en su estrategia de intervención, contempló desde sus inicios la educación como un área estratégica a ser desarrollada. El programa se enfocó en contribuir a resolver tres problemas: 1) Falta de coherencia y/o carencia de una currícula en fitoprotección estructurada y acoplada a las condiciones de las universidades locales y de los centros técnicos agronómicos, 2) poco desarrollo o ausencia de un programa de manejo integrado de plagas ajustado a la práctica de los estudiantes, a la filosofía del Aprender-Haciendo, y 3) un sistema educativo agrícola no acoplado a los nuevos requerimientos que la sociedad y la población rural esperan encontrar en un graduado.

La estrategia de intervención inició con un proceso de consulta con las entidades rectoras, docentes y estudiantes de los centros educativos y un ajuste de prioridades planteadas a través de las demandas de la sociedad en el medio rural. En cada fase se generó un plan de trabajo y una estrategia programática a mediano plazo.

La primera fase se inició con un plan de capacitación para los docentes que impartían las clases de MIP-MIC en temas de relevancia como entomología, malezas, fitopatología, manejo integrado de plagas, manejo integrado de cultivos, generación y desarrollo de tecnología, sistemas de formación profesional, redacción técnica, estadística, formación curricular y Aprender-Haciendo.

En la segunda fase, ya con un grupo de profesionales capacitados en aspectos técnicos y metodológicos, se inició el proceso de formación de grupos especializados conformados por las universidades, en Nicaragua se conformó el Comité Nacional de Enseñanza Agropecuaria (CNEA), y en El Salvador la Comisión de Enseñanza Fitosanitaria de El Salvador (CEFES). Se coordinó y apoyó a los bachilleratos y escuelas de agricultura, y los institutos técnicos como el INATEC en Nicaragua y el INFOP en Honduras. Estos grupos institucionales se formaron para gestionar acciones sobre temas educativos de investigación. Se formularon investigaciones de campo y proyectos con productores en comunidades circundantes a estos centros educativos involucrando a los estudiantes.

Para las fases siguientes, PROMIPAC continuó fortaleciendo las acciones de la CNEA en Nicaragua, la CEFES en El Salvador, y en Honduras a centros de formación técnica y bachilleratos agrícolas liderados por INFOP y el Ministerio de Educación. Toda acción que tuviera que ver con la enseñanza agropecuaria en MIP era coordinada por estos grupos organizados: foros educativos, congresos nacionales $\mathrm{e}$ internacionales de fitoprotección, desarrollo de material educativo y gestión de políticas educativas. Como resultado de este esfuerzo asociativo se formularon guías para fitoprotección y/o agricultura que fueron elaboradas, consensuadas e incluidas en las currículas de las instituciones. PROMIPAC, conjuntamente con miembros de la CNEA, CEFES, el Ministerio de Educación de Honduras e INFOP, desarrollaron en estos esfuerzos de carácter 
interinstitucional, varias series de materiales educativos para formación profesional de calidad y excelencia avalados por editores de renombre. Estos materiales, libros, cartillas, programas radiales, programas televisivos y tutoriales son ampliamente usados por los estudiantes.

Con esta visión de formación por excelencia, donde el Aprender-Haciendo es la filosofía que prevalece, el sector educativo se planteó que para manejar un proceso sostenible de educación constante y de calidad requería la acreditación y formación de instituciones referentes en las temáticas de MIP-MIC, BPA y Doctores en Plantas por eso PROMIPAC formó, acreditó y validó a estos referentes nacionales en la última fase.

Zamorano, como un aporte a la sociedad, en el marco del Programa PROMIPAC, proveyó y desarrolló módulos sobre agua, suelos, fitoprotección, agricultura orgánica y biotecnología, y seis talleres sobre Buenas Prácticas Agrícolas (BPA) en Honduras y Nicaragua. A nivel de estudios avanzados se realizaron tres diplomados a distancia sobre MIP-ECA en Zamorano, dos diplomados BPA en Nicaragua y un diplomado MIP-MIC-agroecología en Nicaragua. Al final del programa se capacitaron en MIP a 729 docentes y 2,382 estudiantes y se realizaron 19 ECA en el tema educativo.

\section{Investigación}

La investigación fue fundamental durante toda la vida del programa como área estratégica generadora y validadora de tecnologías para el manejo fitosanitario de los cultivos, aunque la investigación solo tenía $15 \%$ del presupuesto. PROMIPAC fungió de enlace entre la academia y el campo, mucha investigación se realizó dentro del marco de investigación científica, donde los estudiantes de los centros educativos agrícolas llevaron el liderazgo para desarrollar procesos de investigación en las zonas rurales de influencia del programa. El programa también promovió y lideró procesos de investigación campesina directamente con agricultores líderes.

En la primera fase, el área de investigación se enfocó en el apoyo a las investigaciones para tesis de graduación en los temas de Manejo Integrado de Plagas de estudiantes de las instituciones educativas miembros de la CNEA en Nicaragua y la Escuela Agrícola Panamericana, Zamorano. Se desarrolló un proceso de experimentación campesina desde los grupos regionales en Nicaragua basado en la demanda y la problemática de los grupos de agricultores atendidos.

Durante la segunda fase se apoyó y facilitó el desarrollo de 24 proyectos de investigación ejecutados por socios. Más de 60 materiales, entre informes, notas técnicas, publicaciones en boletines, artículos de revista y libros que contenían resultados de las investigaciones, fueron elaborados. Se acompañó la ejecución de más de 200 experimentos realizados por productores en parcelas de aprendizaje o en sus fincas.

En la tercera y cuarta fase, se desarrollaron procesos de capacitación a facilitadores de las instituciones socias en metodologías de investigación formal y experimentación campesina. En Nicaragua, se logró el establecimiento de un proceso de investigación participativa para mejorar tecnologías que los productores ya utilizan en sus parcelas de producción con el apoyo de universidades como la UNAN-León, la Universidad Católica del Trópico Seco (UCATSE) y la Unión de Cooperativas de Servicios Múltiples (UCOSEMUN) en conjunto con especialistas de la Universidad Nacional Agraria (UNA). Se establecieron experimentos manejados por grupos de agricultores experimentadores como una manera de generar, mejorar y validar tecnologías MIP. En Honduras se apoyaron procesos de investigación formal en el tema de Manejo Integrado de Plagas en hortalizas. También hubo experimentación campesina, lo cual impulsó el encuentro de agricultores experimentadores durante esta fase. En total se realizaron 31 experimentos en Nicaragua y 37 en Honduras. En El Salvador se impulsó la investigación sobre jocote corona, paratrioza en papa y otros rubros, además de capacitar a docentes investigadores en metodologías de investigación.

En la quinta fase, el programa siguió generando tecnologías a través de tesis con estudiantes y experimentaciones con productores líderes. Se continuó fortaleciendo a los facilitadores y productores en metodologías de investigación. Se hizo mucho énfasis en el desarrollo y validación de estructuras de protección de cultivos para el manejo de plagas. Las instituciones socias y los facilitadores 
quedaron dotados de material de referencia ya que se publicó una guía de experimentación. En Nicaragua se formaron grupos de trabajo o redes por cultivos o plagas específicas con el fin de enfocar las prioridades de investigación a los intereses o requerimientos de los productores.

\section{Áreas Transversales}

Monitoreo y evaluación. Para la implementación del sistema de monitoreo y evaluación se tomó como base la experiencia del proyecto MIP Laderas en Honduras. En la primera etapa se determinó el impacto de la capacitación en los productores, en los técnicos y en la institucionalización del MIP. Lo anterior se determinó con grupos de productores y técnicos en instituciones testigos comparando con instituciones socias. También se monitoreó la aceptación del MIP por pequeños productores.

En las fases segunda y tercera, al ser incorporada la estrategia de ECA, el monitoreo y evaluación se orientó en proveer herramientas para medir los resultados de dichos procesos, se levantó la línea base de productores que asistieron a las ECA. En cada ECA se evaluó el conocimiento al inicio y al final, también se documentaron los costos, la producción, el rendimiento y la adopción de prácticas MIP.

En la cuarta fase, se diseñó el monitoreo y evaluación de acciones de desarrollo orientadas al impacto, lo que permitió evaluar los cambios significativos atribuibles a las intervenciones del programa. Además, por ser una metodología participativa, proporcionó un espacio de retroalimentación. En familias capacitadas y no capacitadas se midieron nueve hipótesis de impacto, definidas al inicio del programa, las cuales representan los cambios esperados en las familias beneficiarias durante la duración del mismo.

En la última fase, se incorporaron al sistema de monitoreo las mediciones de impacto económico generado por el programa. Se midió la adopción de las prácticas MIP y se mantuvo el seguimiento a las actividades. Para las mediciones económicas se levantó información de costos e ingresos a través de un registro exacto en bitácoras entregadas a productores participantes del programa y no participantes, para luego comparar la diferencia de las utilidades obtenidas (ingreso neto adicional). Adicionalmente, se midió la utilización de prácticas MIP a través de comparación con línea base y encuestas trimestrales. Los datos muestran, después de la comparación a través del tiempo con un diseño de panel, que las personas atendidas por el programa obtuvieron un ingreso incremental anual estimado de 300 dólares por persona y del total de los productores de granos básicos participantes en el programa, entre el 60 y 76\%, están aplicando prácticas MIP y; del total de productores de hortalizas, el $75-81 \%$ están aplicando dichas prácticas.

Gobernabilidad. El tema de gobernabilidad fue introducido al programa en el 2005 por la Cooperación Suiza. Según el documento - La Gobernabilidad como tema transversal: Guía de orientación para su implementación-, la gobernabilidad comprende procedimientos, políticas y costumbres, que influyen en la manera de administrar, dirigir y controlar una institución. Este concepto incluye también las relaciones entre los actores implicados - gerencias, equipos, socios y beneficiarios - y los objetivos de la institución misma. Los principios de rendición de cuenta, riesgo y eficacia están en el centro de este concepto.

Se incluyó en el Plan Operativo de Fase del programa resultados orientados a mejorar procesos de participación y rendición de cuentas. La elaboración de los planes anuales se realizó de forma conjunta y participativa con los socios. Se implementó un plan de rendición de cuentas y ejecución presupuestaria transparente dentro del programa, cuya valoración de parte de los socios fue $90 \%$.

La gobernabilidad únicamente es posible si existe un equilibrio de oportunidades entre los hombres y las mujeres, así que se promovió el tema de género como parte del proceso de buena gobernabilidad.

Género. La temática de género fue incluida desde los inicios del programa y luego el enfoque se fue mejorando a través de asesorías y capacitaciones al equipo y los socios. En la primera y segunda fase se realizaron por primer vez, investigaciones sobre la participación de la mujer y niños en el manejo integrado de plagas, se visibilizó el trabajo de las mujeres en la agricultura especialmente en el control 
de plagas así como la situación vulnerable que enfrenaban al estar expuestas a plaguicidas. Se trajo a la mesa la temática a través del foro Diálogo Nacional: Género y MIP, se publicó un documento con los resultados del evento.

En las siguientes fases del proyecto se recibió un fuerte acompañamiento de la Cooperación Suiza en el tema de género. Se elaboraron, por primera vez, indicadores medibles y un plan de actividades específicas sobre género. Se capacitó a todo el equipo de PROMIPAC y se asignó una persona responsable para dar seguimiento a la ejecución del plan de actividades. A partir de esta fase, el enfoque se orientó a transversalizar la temática y visibilizar acciones concretas. Se elaboraron módulos de enseñanza en MIP con enfoque de género, se realizaron foros nacionales, se recolectaron y presentaron los datos desagregados por sexo y se incluyeron actividades de sensibilización sobre género en las ECA. En el área de investigación se realizaron tesis para analizar los criterios de adopción del MIP diferenciado por sexo.

Gestión del conocimiento. Fue introducida como área transversal del programa en el 2005. La gestión del conocimiento del programa tuvo dos objetivos, 1) administrar la información generada por el programa y las instituciones socias e 2) impulsar acciones que ayuden a transformar la información generada en conocimientos, en instituciones socias $y$ en agricultores y estudiantes.

En la última fase, el proyecto divulgó oportunamente la información generada en las actividades desarrolladas con los socios, tales como capacitaciones, talleres, publicaciones técnicas, publicaciones metodológicas y sistematizaciones de proceso. Se realizaron encuentros de productores experimentadores para intercambio y establecimiento de redes. Se utilizó el modelo Grupos de Aprendizaje, entre productores promotores.

El programa se desatacó por publicar documentos técnicos y metodológicos dirigidos a estudiantes, docentes, técnicos y agricultores en la temática de manejo de plagas. Se creó una página Web que contiene las publicaciones del programa.

El programa utilizó medios de información masivos, como la radio y la televisión. Se transmitieron más de 350 programas radiales y tres programas de televisión. Se publicaron artículos en revistas de importancia agrícola como Ceiba y LEISA. El programa también produjo información técnica en un diseño de multimedia en los temas de BPA y ECA, y diplomados en línea certificados por Zamorano.

Incidencia en políticas. Esta área tuvo como objetivo incidir en las instancias e instituciones con el fin de promover tecnologías, enfoques y políticas, que al final concluyeran en una producción más sana y sostenible.

En Nicaragua se ayudó a conformar el Comité Nacional de Manejo Integrado de Plagas, el cual estuvo conformado por representantes de instituciones de gobierno y universidades. El objetivo de este comité fue incidir en las políticas para crear conciencia gubernamental y poner restricciones al uso de plaguicidas de alta toxicidad. A través de este comité se impulsaron foros y congresos MIP a nivel de país. También se conformaron grupos regionales de MIP y grupos de especialistas los cuales manejaban acciones, y políticas para sus respectivos sectores. Mediante las acciones de estos grupos y PROMIPAC se logró que las instituciones nacionales reconocieran al MIP como el enfoque oficial del sector agrícola en Nicaragua.

PROMIPAC impulsó el fortalecimiento de la Red de Buenas Prácticas Agrícolas liderada por el MAGFOR. Gracias a esta acción se logró que se diera la discusión y aprobación de leyes y políticas para establecer la certificación de fincas BPA en Nicaragua.

Bajo la incidencia del programa en el sector educativo se actualizaron los pensum los programas agrícolas. El INATEC oficializó estos pensum académicos.

En Honduras, la Secretaría de Educación aprobó la currícula para los bachilleratos agrícolas. PROMIPAC entregó al gobierno 6 libros de texto actualizados para el uso de estudiantes y profesores. El INFOP abrió una nueva carrera en Promotoría Agrícola con el fin de brindar servicios al sector rural y que los promotores agrícolas puedan tener una certificación. Se realizó una compilación de normativas relacionadas al mercado internacional Globalgap.

En El Salvador se conformó la Comisión para la 
Sostenibilidad del MIP presidida por el Viceministerio de Agricultura y Ganadería, y la Cancillería. La Comisión estaba integrada por instituciones estatales, ONG, universidades y bachilleratos técnicos. La Comisión realizó una consulta nacional para determinar las prioridades del MIP a futuro y con estos insumos se preparó una propuesta de proyecto que se sometió a donantes. La Comisión logró una declaración interinstitucional donde se proponía el MIP como estrategia nacional para enfrentar los problemas de plagas.

\section{Sostenibilidad del MIP}

En el sector gobierno, el INTA de Nicaragua adoptó acciones en MIP MIC en sus planes operativos y la utilización de ECA para una campaña masiva de extensión. PROMIPAC, con apoyo de especialistas de Zamorano entrenó y certificó a 221 técnicos del INTA en ECA y un diplomado MIP.

En El Salvador, se formó el Grupo Gestor del Mejoramiento de Educación Agrícola que conformó la CEFES que analizó e incidió en la curricula de los centros educativos del sector agrícola. Se impulsó el establecimiento de un movimiento de instituciones socias con el objetivo de encontrar alternativas de sostenibilidad de la temática MIP, el que estuvo integrado por el Ministerio de Agricultura, Relaciones Exteriores, seis instituciones socias y PROMIPAC. Hoy día la metodología ECA, introducida en el 2000 por PROMIPAC, es utilizada por varias instituciones del sector gobierno y privado.

En Honduras se logró la incorporación de los principios y tecnologías MIP en las políticas institucionales de las instituciones socias, que continuarán promoviéndolas con sus beneficiarios. En la última fase del programa se colaboró con SENASA para actualizar, automatizar y sistematizar toda el área de registros de plaguicidas con el fin que los productores tengan información al día sobre los productos fitosanitarios y veterinarios legalizados en el país. Este sistema será parte permanente del servicio informativo en MIP que la SAG presta a los productores. En educación en Honduras, PROMIPAC institucionalizó en Zamorano los módulos de aprender haciendo en manejo del agua y suelo, y MIP.

\section{Factores que Contribuyeron al Éxito}

\section{De los socios}

1. Se requirió un acompañamiento permanente, dinámico, participativo y de calidad por parte del programa a las instituciones socias, para lograr los resultados deseados.

2. Se realizaron alianzas con instituciones comprometidas que creían que el MIP era una solución alternativa a los problemas fitosanitarios.

3. Se contó con un marco lógico bien estructurado, pero flexible, para adecuar la estrategia de trabajo según las necesidades y diversidad de las instituciones socias.

4. Se logró mayor compromiso de las instituciones socias, en la ejecución de las actividades, al realizar planes operativos participativos.

5. Para la sostenibilidad en la incorporación de temáticas MIP en instituciones educativas se requiere de programas a largo plazo.

6. El enfoque participativo del programa permitió llegar a miles de beneficiarios mediante las acciones de los socios operativos.

\section{De aspectos técnicos}

1. La ampliación del concepto de MIP hacia un concepto más integral de manejo de cultivos permitió incrementar la producción y por lo tanto adopción del MIP.

2. Con la implementación del programa se llenó un vacío temático y tecnológico en la región, por lo que PROMIPAC posicionó el MIP en la misma.

3. MIP/MIC es complejo, tiene muchas variables y requiere de metodologías de extensión como las Escuelas de Campo.

4. Se generaron investigaciones científicas basadas en la demanda de problemas reales a través de un vínculo entre el programa, la academia e instituciones internacionales especializadas.

5. Los problemas fitosanitarios son evolutivos y requieren de procesos constantes de entrenamiento, investigación y generación de tecnologías alternas. 
6. Para tener programas MIP exitosos, teniendo menor incidencia de plagas y enfermedades se requirió pasar de estrategias de control curativo a estrategias de manejo preventivos.

7. Los esfuerzos para reducir el uso de plaguicidas se ven disminuidos por la falta de aplicación de políticas adecuadas.

8. La temática de uso seguro de plaguicidas presenta un fuerte arraigo cultural como obstáculo, por ende, programas MIP que fomentan el uso de plaguicidas alternativos menos tóxicos, contribuyen a reducir contaminación y accidentes laborales.

\section{De la participación institucional de Zamorano y COSUDE:}

1. El liderazgo de Zamorano en agricultura a nivel latinoamericano facilitó la apertura y el posicionamiento del programa.

2. El respaldo institucional de Zamorano a través de los docentes, laboratorios y centros de enseñanza, fortaleció el trabajo técnico de PROMIPAC.

3. La estrategia de intervención a largo plazo de la Cooperación Suiza en América Central permitió diseñar estrategias coherentes.

\section{El Legado de PROMIPAC}

1. La incorporación de las Escuelas de Campo en los sistemas de extensión.

2. El Manejo Integrado de Plagas está institucionalizado en los programas de gobierno y otras instituciones socias.

3. La Carrera de Promotoría Agrícola queda institucionalizada en Nicaragua y Honduras.

4. Establecidas y acreditadas instituciones referentes nacionales en cada una de las temáticas que el programa trabajó.

5. En Zamorano queda una instancia de promoción del MIP por medio de un centro de información, una parcela didáctica para el entrenamiento de agricultura y facilitadores con tecnología para el manejo de problemas fitosanitarios.
6. Aumento del capital humano formado: técnicos, docentes, estudiantes, promotores.

7. Se posicionó a Zamorano y la Cooperación Suiza en el tema MIP.

8. Los productores, con el apoyo técnico, generaron mayores ingresos.

9. Información técnica y metodológica de calidad disponible a través de publicaciones.

10. Creación de grupos especializados que trabajan en MIP.

11. Generación de espacios de análisis y discusión de la temática MIP.

12. Establecida dinámica entre instituciones socias que les permite la discusión de futuras acciones en MIP.

\section{Documentos Consultados}

Comité Nacional de Manejo Integrado de Plagas. 2006. Memoria Foro Taller Implementación de Buenas Practicas Agrícolas y Pecuarias "Estado actual y futuro". Managua, Nicaragua. $28 \mathrm{p}$.

Consorcio EAP, Vecinos Mundiales, CIDICO, Loma Linda Y CRS. 1993. Propuesta Proyecto: Manejo Integrado de Plagas con pequeños productores en Agricultura de Laderas en Honduras. Financiado por el Programa de Naciones Unidas para el Desarrollo (PNUD). Unidad Ejecutora: Escuela Agrícola Panamericana. Tegucigalpa, Honduras. 18 p.

Cooperación Suiza en América Central. 2008. Informe de Fase Programa de Manejo Integrado de Plagas para América Central. Managua, Nicaragua. 43 p.

Escoto, J. A. 2008. Estudio de adopción de tecnologías MIP en Nicaragua y El Salvador. San Salvador, El Salvador. $100 \mathrm{p}$.

Lastres, L. y H. Argüello. 2008. Identificando insectos importantes en la agricultura: un enfoque popular. PROMIPAC, Zamorano, COSUDE. 2da. edición. Carrera de Ciencia y Producción Agropecuaria, Escuela Agrícola Panamericana. Zamorano Academic Press. 90 p.

LEISA Revista de Agroecología. 2003. Aprendiendo con las ECAS. 19(1): 84 p.

Manejo Integrado de Plagas con Pequeños Productores de Granos Básicos en Nicaragua. 1994. Propuesta para la fase II (febrero 1995 a enero 1998) presentado a Cooperación Suiza para el Desarrollo (COSUDE). Ejecutado por Escuela Agrícola Panamericana, Zamorano. Managua, Nicaragua. $21 \mathrm{p}$. 
Rueda et al.: La Contribución de PROMIPAC Zamorano al Manejo Integrado de Plagas en América Central

MIP-Zamorano-COSUDE, Instituto Nicaragüense de Tecnología Agropecuaria INTA, Universidad Nacional Agraria UNA, MIP-CATIE. 1996. Manual de manejo integrado de plagas en el cultivo de frijol. Zamorano Academic Press. Managua, Nicaragua. 75 p.

Programa de Manejo Integrado de Plagas. 2003. Informe Anual PROMIPAC, Zamorano, COSUDE. Managua, Nicaragua. $24 \mathrm{p}$.

Programa de Manejo Integrado de Plagas. 2007. Informe Anual PROMIPAC, Zamorano, COSUDE. Managua, Nicaragua. 24 p.

PROMIPAC, FIDER, CECOTROPIC Y AGROPYME/SWISS CONTAC. 2003. Informe de estudio generación y validación de indicadores de pobreza por beneficiarios de proyectos de manejo integrado de plagas. Estelí, Nicaragua. $53 \mathrm{P}$.

PROMIPAC, Zamorano, COSUDE. 2000. Escuelas de Campo: Guía del Facilitador. San Salvador, El Salvador. 100 p.

PROMIPAC, Zamorano, COSUDE. 2004. Plan Operativo de Fase (POF) 2005-2008. Zamorano, Honduras. 38 p.

PROMIPAC, Zamorano, COSUDE. 2004. Plan Operativo de Fase 2005-2008. Zamorano, Honduras. 40 p.

PROMIPAC, Zamorano, COSUDE. 2004. Sistematización de Experiencias en Manejo Integrado de Plagas y Escuelas de Campo. San Salvador, El Salvador. 73 p.

PROMIPAC, Zamorano, COSUDE. 2007. Memoria Comité Consultivo. Choluteca, Honduras. 10 p.

PROMIPAC. 1999. Informe Anual 1988. Estelí, Nicaragua. $46 \mathrm{p}$.

PROMIPAC. 1999. Memoria taller de evaluación $99 \mathrm{y}$ planificación 2000. Moyogalpa, Isla de Ometepe, Rivas. 64 p.

PROMIPAC. 2002. Informe Técnico Anual junio 2001marzo 2002. Estelí, Nicaragua. 18 p.
PROMIPAC. 2002. Memoria taller de evaluación 2002 y planificación 2003. Montelimar, Nicaragua. 60 p.

Stiefel, J.H. 1993. Manejo Integrado de Plagas en Granos Básicos en Nicaragua. Diagnostico Institucional. Enviromental Management Consultans ECOSENS. Cooperación Suiza para el Desarrollo COSUDE/DEH. Managua, Nicaragua. 34 p.

Trutmann P. y J. Bentley. 2003. Mid Term Evaluation of the Programa Manejo Integrado de Plagas en America Central PROMIPAC. Prepared for the Swiss Agency for Humanitarian Aid and Development Cooperation (SDC). Managua, Nicaragua. 83 p.

Zamorano, COSUDE. 1998. Compartiendo una experiencia de Manejo Integrado de Plagas-PROMIPAC Fase 9598. Managua, Nicaragua. 278 p.

Zamorano, INTA, MAG, MIP-CATIE, UNAN León, Proyecto Pikin Guerrero, CARE, COSUDE. 1998. Manual de manejo integrado de plagas en el cultivo de ajonjolí. Zamorano Academic Press, Managua, Nicaragua. $144 \mathrm{p}$.

Zamorano, UNAN LEON, UNA, CATIE, INTERCOOPERATION, IICA. 1998. Género y Manejo Integrado de Plagas. Zamorano Academic Press. Managua, Nicaragua. 166 p.

Zeiss, M., Espinoza, D., Sanchez, R. y D. Silva. 2000. Informe Final Evaluación de Fase 1998-2001. Programa Manejo Integrado de Plagas con Pequeños Productores de América Central. Estelí, Nicaragua. 156 p.

Recibido para publicación el 14 de febrero de 2013. Aceptado para publicación el 18 de marzo de 2013. 